ORIGINAL ARTICLE

\title{
Changing epidemiology of genital herpes simplex virus infection in Melbourne, Australia, between 1980 and 2003
}

\author{
T Tran, J D Druce, M C Catton, H Kelly, C J Birch
}

Sex Transm Infect 2004;80:277-279. doi: 10.1136/sti.2004.009753

See end of article for authors' affiliations .....................

Correspondence to: T Tran, Victorian Infectious Diseases Reference Laboratory, 10 Wreckyn Street, North Melbourne, 3051, Victoria, Australia; thomas.tran@mh.org.au

Accepted for publication 11 March 2004

\begin{abstract}
Objective: To investigate changes in the proportions of patients infected with genital herpes simplex virus (HSV) types 1 and 2 from 1980 to 2003 in Melbourne, Australia.

Methods: A total of 25372 patients were studied retrospectively. The proportions of HSV-1 and HSV-2 detected in these individuals were analysed by age, sex, and genital site.

Results: In 1980 only $15.8 \%$ of HSV positive genital specimens were HSV-1 compared to $34.9 \%$ in 2003. In 2003 HSV-1 was detected in 77\% of patients aged less than 20 years. Females were more likely to be infected with HSV-1, although the rate of increased detection was more pronounced in males. Except for females over the age of 40 , the trend for the increase in HSV-1 was detected in all age groups. No specific genital site in either sex was associated with the increase.

Conclusions: The proportion of genital HSV-1 has increased in Australian patients, although HSV-2 is still the most common cause of genital infection. Confirmation of HSV type is necessary for optimal patient management.
\end{abstract}

$H$ erpes simplex virus types 1 and 2 (HSV-1 and HSV-2) are the most commonly reported causes of genital ulceration. ${ }^{1}$ While infection is often asymptomatic, ${ }^{23}$ symptomatic cases can range from mild to severe. The natural history of these viruses differs, particularly with respect to the likelihood of recurrence, which has been reported to be five times more likely for HSV-2 than for HSV-1. ${ }^{4}$

Historically HSV-2 has been associated with genital infection, whereas HSV-1 has been associated with oral mucocutaneous disease. However, a change in the epidemiology of genital herpes has been recognised for several years, with studies in the United Kingdom and Europe first identifying this. Between 1978 and 1991 the proportion of HSV-1 associated genital herpes increased in Scotland, ${ }^{56}$ and the percentage of HSV-1 in female anogenital infection has increased in England since 1986. ${ }^{7}$ In Norway, HSV-1 has overtaken HSV-2 as the major cause of genital infection in females and the young, ${ }^{8}$ and a Swedish study has shown the regular occurrence of both virus types in "below the neck" infections. ${ }^{9}$ In the United States, increasing proportions of genital HSV-l affecting mainly females ${ }^{10}$ and college students ${ }^{11}$ have been reported. Similar proportions of genital and oropharyngeal HSV-1 infections have also been reported in sexually active HSV-2 seronegative adults. ${ }^{3}$

Only occasional exceptions to this growing body of evidence have been reported. For example, a study of patients attending a genitourinary medicine clinic in London did not detect the increased rate of genital HSV-I infection reported elsewhere in the United Kingdom, but did observe a wide discrepancy in prevalence of HSV-1 and HSV-2 between ethnic groups. ${ }^{12}$

We report here the results of a retrospective case series examining the relative proportions of HSV-1 and HSV-2 associated genital infection in patients living in Melbourne, Australia, during the period 1980 to 2003. These patients attended general practices, specialised infectious diseases clinics within hospitals, or a large sexual health clinic and had laboratory confirmed HSV genital infections. The relations between infecting HSV type and sex, age at the time of presentation and the anatomical site of infection were investigated.

\section{MATERIALS AND METHODS}

\section{Patients and specimens}

Ethical approval for the study was obtained from the ethics committee of the Royal Melbourne Hospital Research Foundation. Swabs from genital lesions of patients attending general practices, infectious diseases clinics within hospitals, a large sexual health clinic, or specialised infectious diseases clinics were sent to the laboratory for HSV detection. A case was defined as a patient tested between 1980 and 2003 who was positive for HSV-1 or HSV-2 in any of the following genital sites: penis, glans, foreskin, meatus, scrotum, vulva, vagina, clitoris, labia, cervix, introitus, fourchette, prepuce, urethra, anus, perianus, perineum, groin, pubic area, and buttock. For each year between January 1980 and December 2003, data on patient age, sex, genital site of infection, and HSV type identified were analysed. The clinical notes available usually did not indicate whether the lesions present were the result of primary, non-primary first episode, or preexisting infections. However, when sequential specimens were sent only the result of the first specimen was included in the analysis. Only one positive result was included in the data analysis when multiple specimens were sent from the one patient during a clinical episode. Possible risk factors for acquisition of infection in these patients, including numbers of sexual partners, sexual orientation, ethnicity, and socioeconomic status were generally not provided with the test request and could not be included in the analysis.

\section{Laboratory diagnosis}

Between 1980 and 1999 HSV was isolated by inoculating clinical material into human embryonic lung cells which were subsequently examined for the characteristics of HSV specific cytopathic effects (CPE). ${ }^{13}$ The HSV serotype in CPE positive cultures was then confirmed by a neutralisation test incorporating serotype specific antibodies. From late 1999 a multiplex polymerase chain reaction (PCR) capable of detecting HSV-1, HSV-2, cytomegalovirus, and varicella zoster virus was used as the sole method for the diagnosis of HSV infection. ${ }^{14}$ Although this PCR was generally more

Abbreviations: CPE, cytopathic effects; HSV, herpes simplex virus 
sensitive than HSV isolation, both methods were of equivalent specificity for HSV-1 and HSV-2. ${ }^{14}$

\section{Statistical analysis}

Categorical variables were compared using the $\chi^{2}$ distribution. Changes in the proportion of HSV-1 over time were assessed using the $\chi^{2}$ test for trend. Analyses were performed using Epi-Info version 6. ${ }^{15}$

\section{RESULTS}

Proportions of HSV-1 and HSV-2 in genital specimens Data were analysed on a total of 25372 individuals with HSV strains detected in genital sites during the period 1980-2003. Of these, 6462 (25.5\%) were HSV-1 and 18910 (74.5\%) were HSV-2 (table 1). The age range of both males and females included in the study was 1 to 93 years. The sex distribution of individuals from whom HSV positive specimens were obtained is shown in table 1 . The number of HSV strains detected from year to year was relatively constant throughout the period of study (mean 1057, SD 352). In 1980, 15.8\% of all genital infections were caused by HSV-1. This rose to $34.9 \%$ by 2003 ( $\mathrm{p}<0.00001$ ) (fig 1 ).

\section{Sex distribution of genital HSV strains}

Throughout the 23 year study period and in any single year, more laboratory confirmed genital HSV-1 infections were reported in females than males (4755 versus 1707, respectively) (table 1). More females than males also reported confirmed HSV-2 genital infection (10 191 versus 8719, respectively) although in the years 1980-4 more males than females were infected with this serotype (results not shown). Although the temporal increase in genital HSV-1 occurred in both males and females, it was more pronounced in males (fig 1). Between 1980 and 2003 the percentage of males with genital HSV-1 rose from $7.7 \%$ to $28.8 \%$ compared to an increase from $24.7 \%$ to $39.4 \%$ in females. This sex difference was statistically significant $(\mathrm{p}<0.00001)$.

\section{Distribution of genital HSV-1 according to age}

Between 1980 and 2003 the proportion of genital HSV-1 increased for all age groups $(\mathrm{p}<0.00001)$ except those aged 60 years and over (fig 2 ). However, there was a difference by sex in this increase. Whereas the increase occurred for all age groups in males, it was only seen in females up to the age of 40 years (results not shown). People younger than 20 years in 2003 were more likely to be diagnosed with HSV-1 (77.3\% of positive swabs) than HSV-2.

\section{Proportions of HSV-1 and HSV-2 according to genital site}

An analysis of HSV type according to the most common genital sites from which specimens were received was

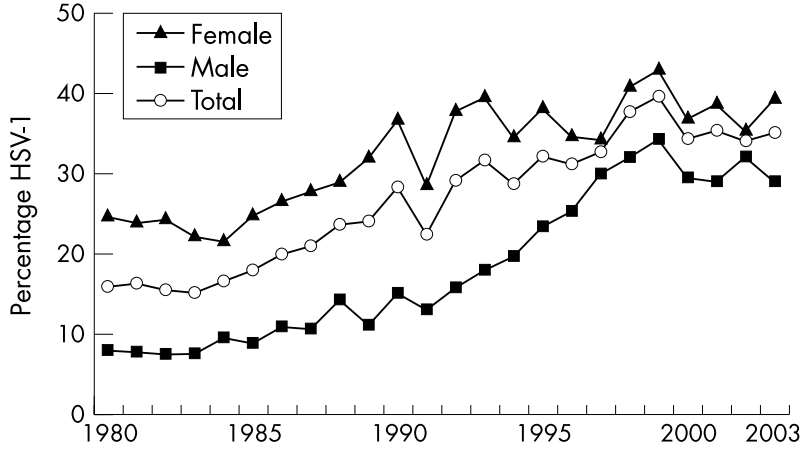

Figure 1 Annual percentage of HSV-1 and HSV-2 detected in genital swabs from males and females between 1980 and 2003.

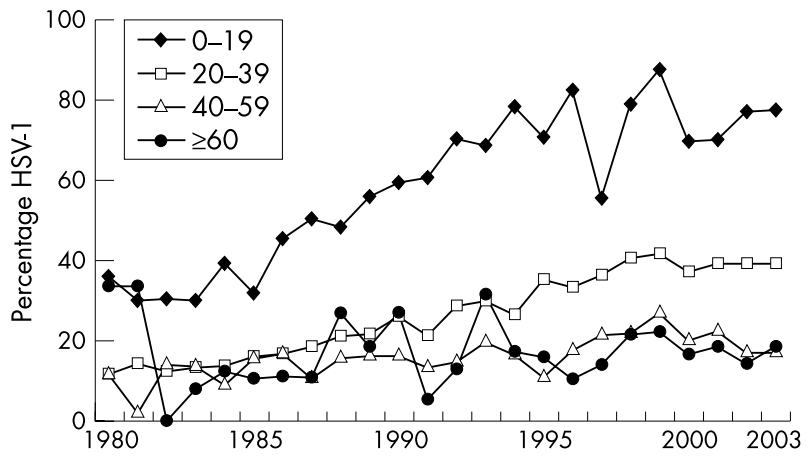

Figure 2 Percentage of HSV-1 in genital sites between 1980 and 2003 according to patient age at the time of specimen collection.

undertaken for both males and females. For males the sites analysed were penile ( $\mathrm{n}=6957$ strains), anal ( $\mathrm{n}=815)$, and buttock ( $\mathrm{n}=374$ ), representing $66.7 \%, 7.8 \%$, and $3.6 \%$, respectively, of all specimens received from males. For females, the sites were vulval $(\mathrm{n}=5985,40 \%)$, labial $(\mathrm{n}=$ $1409,9.4 \%)$, vaginal $(\mathrm{n}=1324,8.9 \%)$, buttock $(\mathrm{n}=1240$, $8.3 \%)$, cervical ( $\mathrm{n}=634,4.2 \%)$, and anal $(\mathrm{n}=480,3.2 \%)$. No statistically significant difference was seen in either males or females in the proportion of HSV-1 in any of these anatomical sites (results not shown).

\section{DISCUSSION}

Our study on patients living in Melbourne, Australia, is the largest and covers the longest time span of any previously reported investigation. In terms of demonstrating an increase in the proportion of genital HSV cases that are HSV-1, its results confirm those from other continents. However, it

Table 1 Numbers, sex, and age distribution of 25372 patients presenting with genital HSV-1 or HSV-2 between 1980 and 2003

\begin{tabular}{lll}
\hline Sex and age (years) of cases & HSV-1 (total (\%)) & HSV-2 (total (\%)) \\
\hline Males & $1707(6.7)$ & $8719(34.4)$ \\
$0-19$ & $147(8.6)$ & $232(2.7)$ \\
$20-39$ & $1298(76.0)$ & $6472(74.1)$ \\
$40-59$ & $227(13.3)$ & $1705(19.6)$ \\
$\geqslant 60$ & $35(2.1)$ & $310(3.6)$ \\
Females & $4755(18.7 \%)$ & $10191(40.2)$ \\
$0-19$ & $1005(21.1)$ & $807(7.9)$ \\
$20-39$ & $3217(67.7)$ & $7508(73.9)$ \\
$40-59$ & $434(9.1)$ & $1525(15.0)$ \\
$\geqslant 60$ & $99(2.1)$ & $351(3.4)$ \\
Total & $6462(25.5 \%)$ & $18910(74.5)$ \\
\hline
\end{tabular}




\section{Key messages}

- The epidemiology of genital HSV infection in Melbourne, Australia, has been changing since at least 1980

- HSV-1 is now the causative agent of approximately one third of genital HSV infections diagnosed in Melbourne

- More than $70 \%$ of people under the age of 20 reporting genital HSV infection are infected with HSV-1

provides new information on the generalised nature of this increase among males and females in most age groups, particularly those under 20 years of age. Based on the number of specimens received over the 23 years analysed, females were more likely to seek treatment than males. Whether this is due to a higher level of symptomatic infection in females could not be ascertained.

The study had some limitations. Because it analysed data gathered over more than 20 years, unrecognised changes in health seeking behaviour in the population studied may have impacted on the results obtained. A change in laboratory identification of HSV from virus isolation to molecular technology occurred in 1999, but because of the equivalent specificity of the two methods this is unlikely to have impacted on the overall conclusions. Also, the laboratory usually received only brief clinical notes, making it difficult to classify some patients as having primary, first presentation, or recurrent infections.

Factors suggested by others to explain the changing epidemiology of HSV genital infection include a reduction in the seroprevalence in childhood of HSV-1 associated with improved hygiene. Evidence exists that immunity resulting from HSV-I infection reduces the likelihood of symptomatic genital HSV-2 infection ${ }^{16}$ and presumably HSV-1 infection. Therefore, one likely manifestation of a reduction in HSV-1 immunity is an increase in symptomatic, laboratory confirmed, HSV-1 genital infection, which will be exacerbated if oral sex practices are also increasing. In the United Kingdom and Holland the prevalence of antibodies to HSV-I has fallen since the late 1980s. ${ }^{17}$ Such information is not available in Australia, but the overall similarities in living standards existing in these countries suggests a similar trend is likely.

Changes in sexual practices linked with concerns about safe sex may also contribute to the changing epidemiology of this infection. The reasons for these changes are varied but may include that unwanted pregnancies can be avoided and the perception, particularly among teenagers, that sexually transmitted diseases are not transmitted by oral sex. ${ }^{18}$ Receptive oral sex within the previous 2 months has been associated with an increased likelihood that the initial infection was HSV-1. ${ }^{19}$ Despite the apparent increase in orogenital sex seen in many communities, an increased proportion of HSV-2 in orolabial lesions has not been detected in our study (results not shown) or studies undertaken by others. ${ }^{20}$

At this time in Melbourne, HSV-1 is more common than HSV-2 in patients under 20 years of age and the likelihood that females will have an HSV-1 genital infection is now similar to that for HSV-2. Patients with genital HSV-1 are likely to have different clinical outcomes compared to patients with HSV-2 infection. The natural history of HSV-I infection is relatively mild, particularly regarding the likelihood of recurrence, which has been estimated to be approximately one fifth that of HSV-2 during the first year after primary infection. ${ }^{4}$ This may influence the information given to patients at the time of diagnosis, including whether suppressive or episodic antiviral therapy may be required in future. Vaccine strategies designed to provide immunity or ameliorate the clinical symptoms associated with HSV infection must also account for the now widespread occurrence of both HSV-1 and HSV-2 in this condition.

\section{ACKNOWLEDGEMENTS}

We thank Kirsty Minerds, Bill Maskill, and the staff of the virus detection laboratory over many years for their assistance with data collection and analysis.

\section{CONTRIBUTORS}

TT, medical scientist performing laboratory testing, data analysis, and manuscript preparation; JDD, senior medical scientist involved in laboratory test design and performance, and review of the manuscript; MCC, medical virologist and review of the manuscript; HK, epidemiologist and statistical advice; CJB, senior medical scientist with responsibility for test results, data analysis, and manuscript review.

\section{Authors' affiliations}

T Tran, J D Druce, M C Catton, H Kelly, C J Birch, Victorian Infectious Diseases Reference Laboratory, 10 Wreckyn Street, North Melbourne 3051, Victoria, Australia

\section{REFERENCES}

1 Mertz GJ. Genital herpes simplex virus infections. Med Clin N Am 1990;74:1433-54.

2 Wald A. New therapies and prevention strategies for genital herpes. Clin Infect Dis 1999;28(Suppl 1):S4-13.

3 Langenberg AGM, Corey L, Ashley RL, et al. A prospective study of new infections with herpes simplex virus type 1 and type 2. N Engl J Med 1999;341:1432-8

4 Engelberg R, Carrell D, Krantz E, et al. Natural history of genital herpes simplex virus type 1 infection. Sex Transm Dis 2003;30:174-7.

5 Ross JDC, Smith IW, Elton RA. The epidemiology of herpes simplex types 1 and 2 infection of the genital tract in Edinburgh 1978-1991. Genitourin Med 1993;69:381-3.

6 Scoular A, Leask BGS, Carrington D. Changing trends in genital herpes due to herpes simplex virus type 1 in Glasgow, 1985-88. Genitourin Med 1990:66:226

7 Tayal SC, Pattman RS. High prevalence of herpes simplex virus type 1 in female anogenital herpes simplex in Newcastle upon Tyne 1983-92. Int J STD AIDS 1994;5:359-61.

8 Nilsen $\mathrm{N}$, Myrmel $\mathrm{H}$. Changing trends in genital herpes simplex virus infection in Bergen, Norway. Acta Obstetr Gynecol Scand 2000;79:693-6.

9 Lowhagen G-B, Tunback P, Bergstrom T. Proportion of herpes simplex virus (HSV) type 1 and type 2 among genital and extragenital HSV isolates. Acto Derm Venereol 2002;82:118-20.

10 Ribes JA, Steele AD, Seabolt JP, et al. Six-year study of the incidence of herpes in genital and nongenital cultures in a Central Kentucky medical centre patient population. J Clin Microbiol 2002;39:3321-5.

11 Roberts CM, Pfister JR, Spear SJ. Increasing proportion of herpes simplex virus type 1 as a cause of genital herpes infection in college students. Sex Transm Dis 2003:30:797-800.

12 Strutt M, Bailey J, Tenant-Flowers $M$, et al. Ethnic variation in type of genital herpes simplex virus infection in a South London genitourinary medicine clinic. $J$ Med Virol 2003;69:108-10.

13 Birch CJ, Tyssen DP, Tachedjian G, et al. Clinical effects and in vitro studies of trifluorothymidine combined with interferon- $\alpha$ for treatment of drug-resistant and -sensitive herpes simplex virus infections. J Infect Dis 1992;166:108-12.

14 Druce J, Catton M, Chibo D, et al. Utility of a multiplex PCR assay for detecting herpesvirus DNA in clinical samples. J Clin Microbiol 2002;40:1728-32.

15 Dean AG, Dean JA, Coulombier D, et al. Epi Info, Version 6: a word processing, database, and statistics program for epidemiology on microcomputers. Atlanta, GA: Centers for Disease Control and Prevention, 1994.

16 Xu F, Schillinger JA, Sternberg MR, et al. Seroprevalence and coinfection with herpes simplex virus type 1 and type 2 in the United States, 1988-1994. $J$ Infect Dis 2002;185:1019-24.

17 Cowan FM. Commentary: developing preventative strategies in Europe. Int J Epidemiol 2001;30:588-9.

18 Samra Z, Scherf E, Dan M. Herpes simplex virus type 1 is the prevailing cause of genital herpes in the Tel Aviv area, Israel. Sex Transm Dis 2003;30:794-6.

19 Lafferty WE, Downey L, Celum C, et al. Herpes simplex virus type 1 as a cause of genital herpes: impact on surveillance and prevention. $J$ Infect Dis 2000;181:1454-7.

20 Lippelt L, Braun RW, Kuhn JH. Genital herpes simplex virus type 1 infection: new field for an old acquaintance? Intervirology 2002;45:2-5. 